\title{
Sequential Bilateral Hip Fractures in Elderly Patients
}

\author{
Seong-Hwan Woo, MD, Kyung-Soon Park, MD, Ik-Sun Choi, MD, \\ Young-Sub Ahn, MD, Dong-Min Jeong, MD, Taek-Rim Yoon, MD \\ Department of Orthopedic Surgery, Center for Joint Disease, \\ Chonnam National University Hwasun Hospital, Hwasun, Korea
}

Purpose: To evaluate the incidence and presentation of osteoporotic sequential bilateral hip fractures (SBHF) in Center for Joint Disease, Chonnam National University Hwasun Hospital as there are limited studies with variable results reported in Korea.

Materials and Methods: Records of 507 patients aged >60 years old presenting with osteoporotic hip fractures between 2009 and 2015 were retrospectively reviewed to document the occurrence and presentation of sequential hip fractures; mean post-treatment follow-up was 48 months. Additionally, any correlations between sequential fractures and initial fracture and risk factors were assessed. Bone mineral density (BMD) was measured before and after sequential hip fracture for comparison.

Results: There were 246 femoral neck (Group A) and 261 intertrochanteric (Group B) fractures. The cumulative incidence of SBHF was 8.2\% (42 patients total; 29 in Group A and 13 in Group B). Average interval of SBHF for Group A and Group B were 37.4 months and 29.9 months, respectively. There was significant correlation between the initial fracture type and sequential fractures, particularly the trochanteric and subgroup of those with neck fractures. Hypertension as a co-morbidity and female sex have been identified as risk factors for SBHF. No significant findings were noted regarding BMD and risk factors in both groups.

Conclusion: The clinical presentations of SBHF noted here concur with other worldwide studies and may guide efforts to develop relevant programs to prevent SBHF.

Key Words: Hip fractures, Aged, Osteoporosis, Incidence, Risk factors

Submitted: September 23, 2019 1st revision: November 6, 2019 Final acceptance: December 5, 2019

Address reprint request to

Taek-Rim Yoon, MD

(https://orcid.org/0000-0001-8282-1723)

Department of Orthopedic Surgery, Center for Joint Disease, Chonnam National University Hwasun Hospital, 322 Seoyang-ro, Hwasun-eup, Hwasun 58128, Korea

TEL: +82-61-379-7676 FAX: +82-61-379-7681

E-mail: tryoondachonnam.ac.kr

This is an Open Access article distributed under the terms of the Creative Commons Attribution Non-Commercial License (http://creativecommons. org/licenses/by-nc/4.0) which permits unrestricted non-commercial use, distribution, and reproduction in any medium, provided the original work is properly cited.

\section{INTRODUCTION}

Sequential bilateral hip fractures (SBHF) have been reported with increasing frequency in elderly and osteoporotic patients ${ }^{1}$. Patients with SBHF have greater mortality, reduced functional abilities, limited mobility and seldom recover to their preoperative mobility levels ${ }^{2-4}$, therefore, it is crucial to fully understand SBHF, so that preventive and treatment strategies can be developed and initiated after an initial hip fracture, especially in elderly patients.

Numerous studies have described the clinical presentation and outcomes of SBHF, however, to date, studies of incidence and presentation of SBHF in Korea are scarce $(n=3)$ and results are variable ${ }^{5-7)}$. To help fill this knowledge gap, this study was conducted to investigate the following: (i) 
incidence of SBHF over 7 years in 507 patients with osteoporotic hip fractures; (ii) the presentation of SBHF, its relation to the first fracture, mortality and percentage of patients regaining their pre-injury functional state; and (iii) potential risk factors of SBHF (e.g., age, sex, co-morbidities, anti-osteoporosis treatments, bone mineral density [BMD]).

\section{MATERIALS AND METHODS}

This study was approved by the institutional review board of Chonnam National University Hwasun Hospital, and written informed consent was obtained from all participants (CNUHH-2016-113).

A total of 507 patients with osteoporotic hip fractures caused by low-energy trauma between January 2009 and December 2015 were retrospectively reviewed after institutional ethical committee approval. Patients under the age of 60 at the time of fracture, those with fractures caused by high-energy trauma (e.g., vehicular accidents, falls from height), pathologic fractures caused by cancer metastasis or any other hereditary factors that could affect bony metabolism, were excluded.

Based on the type of initial hip fracture, patients were divided into two groups: (i) neck fracture (Group A), and (ii) intertrochanteric fracture (Group B). All patients were followed-up at 2 weeks, 1, 3 6, 12 months postoperatively and yearly thereafter. Plain radiographs (hip antero-posterior and lateral view) were obtained at every visit. Each patient who did not visit the clinic was surveyed via telephone to investigate postoperative mortality, survival rate, and functional activities.

All sequential hip fractures were documented along with presentation and treatment. Restoration of the preinjury functional activity of sequential hip fractures was assessed. Classifications used were: (i) outdoor activity with an ability to ambulate greater than 5 blocks, (ii) outdoor activity with ambulation fewer than 5 blocks, (iii) outdoor activity with support, (iv) indoor activity, (v) indoor activity with support, (vi) wheelchair ambulation and (vii) bedridden state.

The mortality and co-morbidities of the sequential hip fractures were also evaluated. Patients were followed-up as scheduled; 91 patients who did not return to the hospital by the last follow-up were surveyed via telephone. Deaths were classified according to the time periods: (i) within 3 months and (ii) between 3 months to 1 year. The incidence of co-morbidities (i.e., hypertension, diabetes mellitus, myocardial infarction, cerebrovascular accidents, liver disease, chronic kidney disease) were investigated. In addition, the timeframe of osteoporosis medication initiation after the primary hip fracture and compliance were evaluated.

Changes in BMD between Groups A and B were evaluated when a contralateral hip fracture occurred at intervals of 12 months. The BMD of those with SBHF were evaluated using preoperative $\mathrm{T}$-scores of the lumbar spine, femoral neck, trochanteric area, and total femoral BMD. For primary fractures, the lumbar spine, contralateral femoral neck, trochanter and total proximal femur BMD were used. For secondary fractures, only the lumbar spine BMD was evaluated.

All data are presented as mean, standard deviation and range. IBM SPSS for Windows ver. 20.0 SPSS ${ }^{\circledR}$ software (IBM Corp., Armonk, NY, USA) was used for statistical analysis. The chi-square test was performed to compare the 3-month and within 1-year mortality rates between unilateral and SBHF groups. Significance was set at $P<0.05$. Correlation coefficient was evaluated for initial and secondary fractures, and sub-classification of the fracture patterns. Multivariable logistics regression analysis was used to evaluate potential risk factors of SBHF.

\section{RESULTS}

The patient population included 173 male and 334 female patients with an average follow-up duration of $48.0 \pm 11.1$ months (range, 11.0-82.4 months), mean age of 76.2 \pm 7.5 years (range, 60-100 years) and mean body mass index of $20.9 \pm 3.6 \mathrm{~kg} / \mathrm{m}^{2}$ (range, $16.32-30.4 \mathrm{~kg} / \mathrm{m}^{2}$ ). Out of the 507 patients, there were 246 with femoral neck fractures (Group A) and 261 with intertrochanteric fractures (Group B). The operation methods for hip fractures varied and included internal fixation using compressive hip screw, angled blade plate, intramedullary nailing, bipolar hemiarthroplasty, and total hip arthroplasty. In Group A, arthroplasty was performed in 230 patients and osteosynthesis in 16 patients. In Group B, arthroplasty was performed in 90 patients and osteosynthesis in 171 patients.

A total of 42 out of the 507 patients $(8.23 \%)$ had sequential hip fractures; 29 (11.8\%) and 13 (5.0\%) in Groups A and $\mathrm{B}$, respectively. The mean age was 75.8 years at the time of the secondary fracture. Average interval from initial to sequential hip fracture was 35.1 \pm 32.2 months (range, 0.9116.4 months) and this was further divided into 37.4 months (range, 0.9-116.4 months) in Group A, and 29.9 months (range, 1.5-69.7 months) in Group B.

Out of the 29 patients with sequential fractures in Group 


\section{Hip \& Pelvis}

Seong-Hwan Woo et al. Sequential Bilateral Hip Fractures in Elderly Patients

A, $21(72.4 \%)$ had a femoral neck fracture (Fig. 1) and 8 (27.6\%) had a sequential intertrochanteric fracture. In Group B, there were 12 patients $(92.3 \%)$ with sequential intertrochanteric fractures (Fig. 2) and 1 (7.7\%) suffered from a sequential femoral neck fracture. The correlation between initial fracture to sequential fracture (correlation between femoral neck fracture to femoral neck fracture and intertrochanteric fracture to intertrochanteric fracture), was $\mathrm{r}=0.599(P<0.01)$ (Table 1).

Restoration to pre-injury activity was evaluated; results are presented in Table 2. Twelve out of the 26 patients returned to outdoor activity with more than 5 blocks after the first operation, only $2(16.7 \%)$ returned to outdoor activity with more than 5 blocks after the second operation. The correlation between restoration of pre-injury activity level and mortality rate cannot be evaluated because of its relatively small sample size.

We used multivariable logistics regression analysis to test factors that could serve as independent predictors for SBHF. Female sex (odds ratio $[\mathrm{OR}]=1.920 ; P<0.05$ ) and hypertension $(\mathrm{OR}=1.751 ; P<0.05)$ emerged as independent factors predicting higher risk of SBHF. Osteoporosis
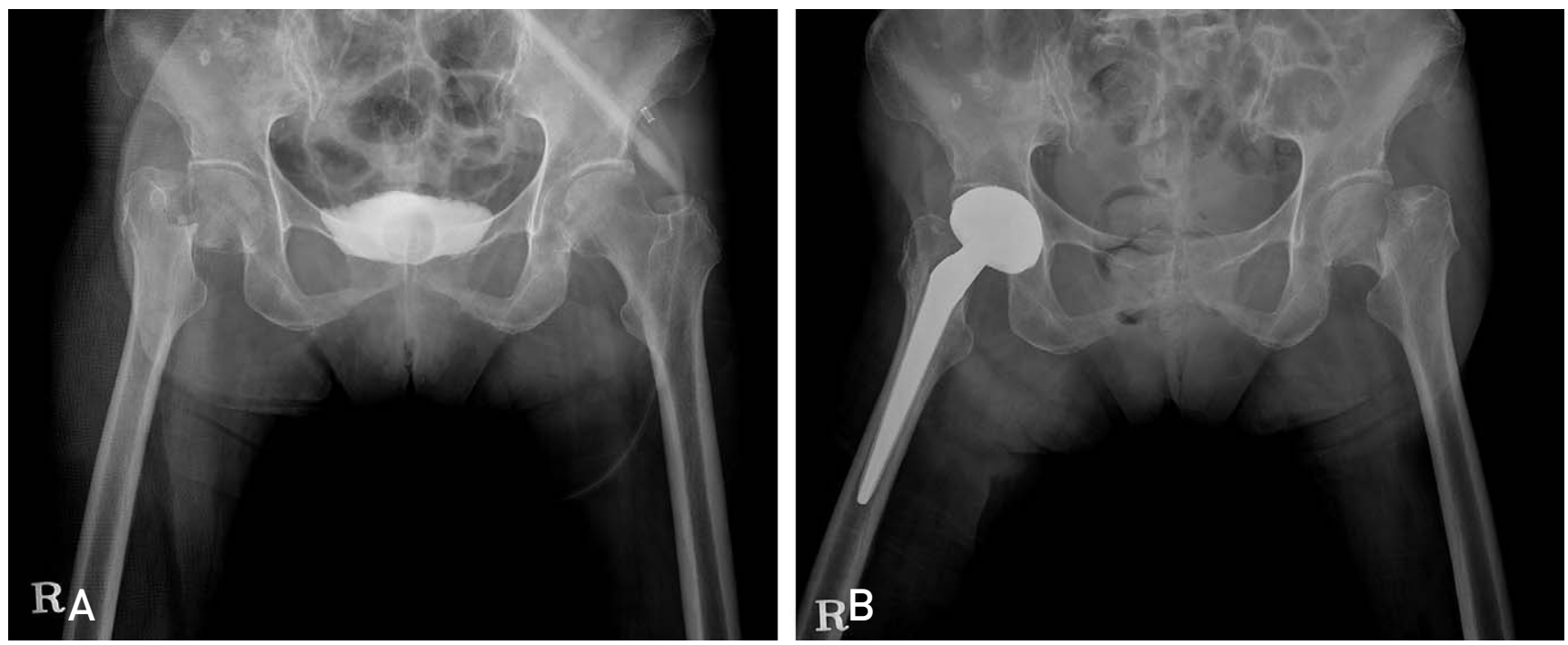

Fig. 1. A 72-year-old lady suffered fracture of her right femoral neck after a trivial fall (A) and was treated with total hip arthroplasty. Seven months later, she had fracture at the contralateral neck of femur when she had another trivial fall (B).
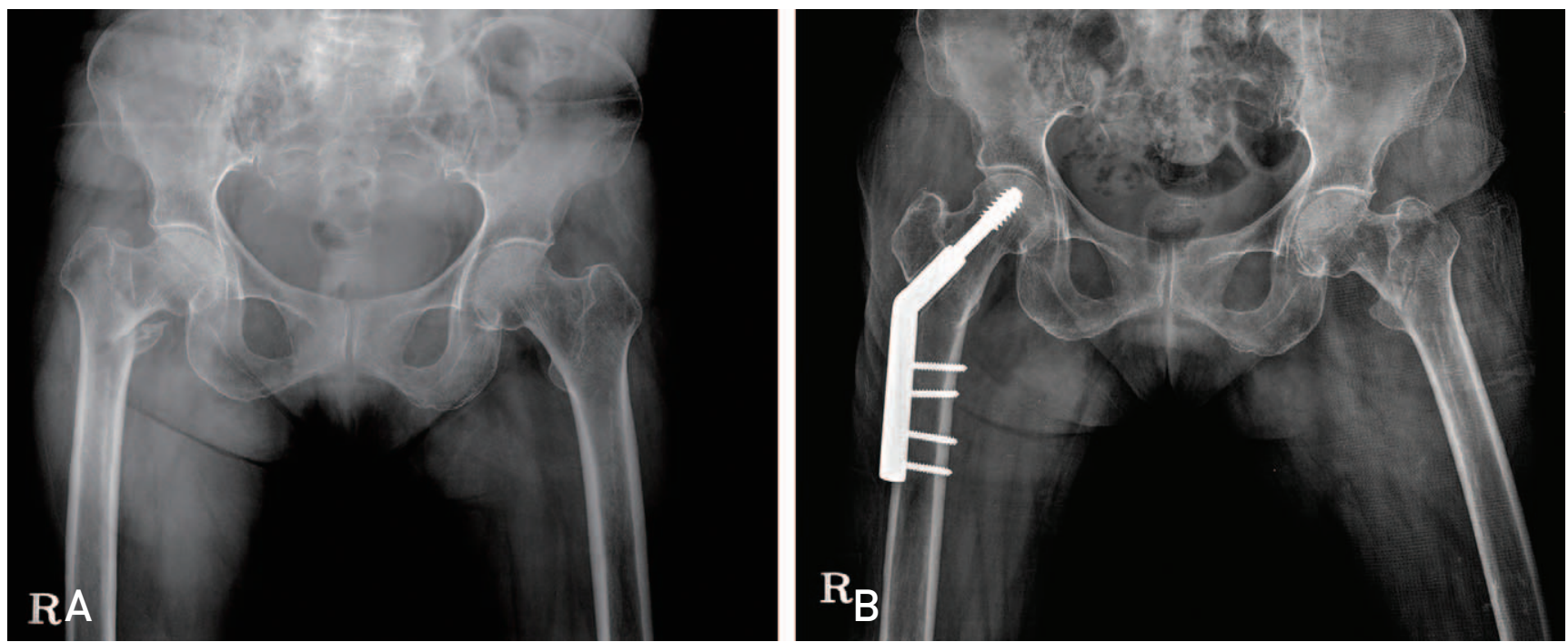

Fig. 2. An 80-year-old lady suffered an intertrochanteric fracture of her right femur after a trivial fall (A). She was treated with compression hip screw. She was well for the subsequent three years, until one day she had another trivial fall. Due to the fall, she had a similar fracture at the contralateral intertrochanteric region (B). 


\section{Hip \& Pelvis}

Hip Pelvis 32(2): 99-104, 2020

Table 1. Correlation between Initial Fx to Sequential Fx in 42 Patients of SBHF

\begin{tabular}{lccc}
\hline \hline Variable & $\begin{array}{c}\text { 1st femoral neck Fx } \\
\text { (total 246, SBHF 21) }\end{array}$ & $\begin{array}{c}\text { 1st intertrochanteric Fx } \\
\text { (total 261, SBHF 13) }\end{array}$ & $P$-value/r* \\
\hline 2nd femoral neck Fx & $21(72.4)$ & $1(7.7)$ & $12(92.3)$ \\
2nd intertrochanteric Fx & $8(27.6)$ & $12.01 / 0.599$ \\
\hline
\end{tabular}

Values are presented as number (\%).

Fx: fracture, SBHF: sequential bilateral hip fractures.

* Coefficient of correlation.

Table 2. Changes of Activity Level in Patients with Sequential Bilateral Hip Fracture

\begin{tabular}{lcrr}
\hline \hline Variable & Pre-injury & After 1st operation & After 2nd operation \\
\hline Outdoor activity more than 5 blocks* & 26 & 12 & 2 \\
Outdoor activity less than 5 blocks & 8 & 5 & 2 \\
Outdoor activity with support & 6 & 17 & 19 \\
Indoor activity & 2 & 5 & 10 \\
Bed ridden state & 0 & 3 & 9 \\
\hline
\end{tabular}

* $500 \mathrm{~m}$.

Table 3. Multivariable Logistic Regression Analysis for Sequential Bilateral Hip Fracture

\begin{tabular}{|c|c|c|c|c|c|}
\hline \multirow{2}{*}{ Variable } & \multirow{2}{*}{ Unilateral ( $n=465$ ) } & \multirow{2}{*}{ Bilateral (n=42) } & \multicolumn{3}{|c|}{ Sequential bilateral hip fracture } \\
\hline & & & OR & $95 \% \mathrm{Cl}$ & $P$-value \\
\hline Sex & $301(64.7)$ & $32(76.2)$ & 1.920 & $0.916-4.028$ & 0.034 \\
\hline Age ( $\geq 75$ yr) & $283(60.9)$ & $26(61.9)$ & 1.161 & $0.610-2.211$ & 0.650 \\
\hline Osteoporosis medication & $187(40.2)$ & $15(35.7)$ & 0.551 & $0.207-1.465$ & 0.132 \\
\hline Hypertension & $177(38.1)$ & $24(57.1)$ & 1.751 & $0.925-3.315$ & 0.045 \\
\hline Diabetes mellitus & $86(18.5)$ & 14 (33.3) & 1.445 & $0.693-3.010$ & 0.326 \\
\hline Myocardial infarction & $22(4.7)$ & $4(9.5)$ & 1.571 & $0.505-4.882$ & 0.135 \\
\hline Cerebrovascular accidents & 32 (6.9) & 5 (11.9) & 1.486 & $0.541-4.080$ & 0.242 \\
\hline Liver disease & $15(3.2)$ & $2(4.8)$ & 1.200 & $0.262-5.504$ & 0.814 \\
\hline Chronic kidney disease & $21(4.5)$ & $4(9.5)$ & 1.387 & $0.440-4.376$ & 0.577 \\
\hline
\end{tabular}

Values are presented as number (\%).

OR: odds ratio, $\mathrm{Cl}$ : confidence interval.

Table 4. Mortality in Patients with Sequential Hip Fracture

\begin{tabular}{lcc}
\hline \hline Variable & No. of patient & $P$-value \\
\hline Death within 3 months & $20 / 507(3.9)$ & \\
Death within 1 year & $62 / 507(12.2)$ & \\
$\quad$ Unilateral & $56 / 465(12.0)$ & 0.633 \\
Bilateral sequential & $6 / 42(14.3)$ & \\
\hline
\end{tabular}

Values are presented as number/total number $(\%)$.

medication reduced the incidence of SBHF, however, there was no statistical significance (OR=0.551; $P=0.132$ ). None of the other variables tested (e.g., age, co-morbidities) were predictive of SBHF (Table 3). It is also noted that diseases (e.g., Parkinson's disease, dementia, rheumatoid arthritis), that can affect postoperative rehabilitation were uncommon in this study, especially in those patients with SBHF. Only 3 patients who suffered SBHF had underlying Parkinson's disease and there were no cases of dementia or rheumatoid arthritis in the SBHF group.

As shown in Table 4, the overall 1-year mortality rate of the 507 patients was $12.2 \%(n=62) ; 20$ patients $(3.9 \%)$ died within 3 months and $42(8.3 \%)$ between 3 months and 1 year; 56 of the 465 patients $(12.0 \%)$ with unilateral and 6 out of $42(14.3 \%)$ with SBHF passed away during the study period, however, no significant difference between the two groups was observed $(P=0.633)$. The causes of death in the SBHF group varied from malignant bowel obstruction $(n=2)$, aspiration pneumonia $(n=2)$ and septic 
$\operatorname{shock}(n=2)$.

All 42 patients in the SBHF group were studied for potential improvement in BMD and use of osteoporosis medications. In Group A, the BMD of the neck area decreased from -2.63 to -2.83 , however, for the trochanteric area, BMD increased from -2.04 to -1.57 . In Group B, there were detrimental changes in BMD in the trochanteric area (from -2.30 to -2.70 ). however, this value increased (from -2.68 to -2.45$)$ in the neck region. The starting rate of osteoporotic medication after primary fracture was $35.7 \%$ (15 out of 42 cases), with 13 cases for medication ( 5 for risedronate, 8 for alendronate) and 2 cases for intravenous bisphosphonate injection (zolendronate). The remaining 27 cases (64.3\%) did not initiate osteoporotic medication due to insufficiency of education, low compliance of the patients, and/or prohibitive cost.

\section{DISCUSSION}

As populations age, the number of osteoporotic hip fractures have markedly increased. It is estimated that in 2050, approximately $50 \%$ of all hip fractures in the world would occur in Asia $^{8}$. Ha et al. ${ }^{9)}$ revealed that: (i) the incidence of hip fracture is increasing in Korea, and (ii) by 2025 , the number of hip fractures will increase by 1.4 fold. Additionally, the socioeconomic burden of hip fracture is expected to rise as well.

It is reported that patients who suffered a hip fracture have an increased risk of experiencing sequential contralateral hip fracture with the reported overall incidence of $8.54 \%$ (range, 5-10\% $)^{1,10,11)}$. The cumulative incidence of SBHF in our study was $8.2 \%$, which concurs with incidences published worldwide and is consistent with the incidence of $8.6 \%$ reported by Lee et al. ${ }^{7}$. However, it is relatively higher than the incidence reported in two other Korean studies (i.e., $4.3 \%$ and $5.5 \%)^{5,6)}$. The exact reason for the difference could not be determined, however, it may be due to the compliance to the osteoporotic medication as higher rate of SBHF occurs in patients who are not compliant to osteoporotic medication ${ }^{7}$.

In this case series, the average time interval to development of a second hip fracture after the first hip fracture was 35.1 \pm 32.2 months (range, 0.9-116.4 months). In other words, more than a half of SBHF $(60.8 \%)$ occurred within 3 years after the first hip fracture. It was observed in this case series that occurrence of SBHF in trochanteric region occurs relatively earlier than SBHF in neck region (29.9 months vs. 37.4 months) although this difference was not statistically significant.

The mortality rate in the current study mirrors results in published literature (i.e., cumulative mortality rate ranging from $12 \%$ to $14 \%)^{1}$. Patients in this study had a slightly higher mortality in cases of bilateral sequential hip fracture than that of the unilateral hip fracture group, even though the difference found was insignificant $(12.0 \%$ vs. $14.3 \%$, $P=0.052)$. The variance could be attributed to longer bed-ridden or inadequate recovery of the activity level postoperation in SBHF group. Results are not conclusive due to small number of subjects but it would suggest that shorter bed-ridden and early recovery of the activity level could be beneficial to reduce mortality rate.

Numerous studies have mentioned the symmetrical presentation of SBHF, namely the same location in the second contralateral hip fracture as the previous fracture. The exact mechanism for this scenario is unclear and explanations put forth include patient's personal gait and bone architectures, symmetrization of morphological criteria, and endogenous criteria ${ }^{10,12,13)}$. This study found strong positive correlations between primary and secondary fracture hip fracture patterns $(\mathrm{r}=0.559, P<0.01, \mathrm{n}=42)$ as reported in other studies ${ }^{14-16)}$. Further analysis revealed that strong positive correlations were present in sub-fracture patterns of femoral neck fractures $(\mathrm{r}=0.775, P=0.03, \mathrm{n}=21)$. Positive correlations were also observed in sub-fracture patterns of intertrochanteric fractures, however, statistical significance was not obtained $(\mathrm{r}=0.169, P=0.599, \mathrm{n}=12)$. The results, although inconclusive of the exact pathophysiology, can be used for patient education and counseling regarding risk prevention, to estimate future fracture, and to prepare operation tools based on SBHF correlations.

Regarding the comparison of potential risk factors between single fractures with SBHF, no strong correlation was established between comorbidities and SBHF. Out of the 42 patients, only $35.7 \%$ were prescribed osteoporotic medication, which was mainly anti-resorptive agents. Many studies have emphasized the importance of starting routine osteoporotic medications to improve BMD and prevent subsequent fractures. Although the prescription and compliance rate in this case series was higher than the rate reported in other studies (i.e., 18.9\%), we strongly believe and recommend medical education about the significance of osteoporosis, the timing of initiating medication, and importance of compliance in the prevention of SBHF. BMD was also measured in this study, as it is the most important quantitative criteria for extent of osteoporosis and could potentially be used to predict a contralateral hip fracture. 
Our findings did not reveal any significant correlation between contralateral fractures and BMD, possibly because of the relatively small sample size.

This study had certain limitations. First, it was a retrospective review and might be subject to unavoidable recall bias, which potentially affects the magnitudes. Second, the number of patients with SBHF is relatively small, thus it was difficult to establish strong correlations. For instance, no correlation was observed between use of osteoporotic medication and fracture risk as only $35.7 \%$ patients had good compliance with their medications. Lastly, the assessment of osteoporosis status improvement with the use of dualenergy X-ray absorptiometry is imperfect and cannot accurately describe the relationship between BMD and fracture patterns; it is believed that better results could be achieved by using better measurement tools (e.g., quantitative computer tomography).

\section{CONCLUSION}

The results of this study demonstrate that the incidence and presentation of SBHF in Korea are similar to previously published worldwide reports. The information shared here increase our current understanding of this fracture type and provide useful information on preventive and treatment strategies to be considered following an initial osteoporotic hip fracture in elderly patients.

\section{CONFLICT OF INTEREST}

The authors declare that there is no potential conflict of interest relevant to this article.

\section{REFERENCES}

1. Zhu Y, Chen W, Sun T, Zhang Q, Liu S, Zhang Y. Epidemiological characteristics and outcome in elderly patients sustaining non-simultaneous bilateral hip fracture: a systematic review and meta-analysis. Geriatr Gerontol Int. 2015;15:11-8.

2. Pearse EO, Redfern DJ, Sinha M, Edge AJ. Outcome following a second hip fracture. Injury. 2003;34:518-21.

3. Sawalha S, Parker MJ. Characteristics and outcome in patients sustaining a second contralateral fracture of the hip. J Bone Joint Surg Br. 2012;94:102-6.

4. Galois L, Dausse Y, Mainard D, Delagoutte JP. Bilateral noncontemporary hip fractures. 31 cases reported. Eur J Orthop Surg Traumatol. 2001;11:219-23.

5. Kim SM, Moon YW, Lim SJ, et al. Prediction of survival, second fracture, and functional recovery following the first hip fracture surgery in elderly patients. Bone. 2012;50: 1343-50.

6. Chang JD, Yoo JH, Reddy P, Lee SS, Hwang JH, Kim TY. Risk factors for contra-lateral hip fracture in elderly patients with previous hip fracture. Injury. 2013;44:1930-3.

7.Lee YK, Ha YC, Yoon BH, Koo KH. Incidence of second hip fracture and compliant use of bisphosphonate. Osteoporos Int. 2013;24:2099-104.

8. Cooper C, Campion G, Melton LJ 3rd. Hip fractures in the elderly: a world-wide projection. Osteoporos Int. 1992;2:285-9.

9. Ha YC, Kim TY, Lee A, et al. Current trends and future projections of hip fracture in South Korea using nationwide claims data. Osteoporos Int. 2016;27:2603-9.

10. Shabat S, Gepstein R, Mann G, Kish B, Fredman B, Nyska $\mathrm{M}$. The second hip fracture: an analysis of 84 elderly patients. J Orthop Trauma. 2003;17:613-7.

11. Gaumetou E, Zilber S, Hernigou P. Non-simultaneous bilateral hip fracture: epidemiologic study of 241 hip fractures. Orthop Traumatol Surg Res. 2011;97:22-7.

12. Fukushima T, Sudo A, Uchida A. Bilateral hip fractures. $J$ Orthop Sci. 2006;11:435-8.

13. Schrøder HM, Petersen KK, Erlandsen M. Occurrence and incidence of the second hip fracture. Clin Orthop Relat Res. 1993;(289):166-9.

14. Yamanashi A, Yamazaki K, Kanamori M, et al. Assessment of risk factors for second hip fractures in Japanese elderly. Osteoporos Int. 2005; 16:1239-46.

15. Saxena P, Shankar J. Contralateral hip fractures: can predisposing factors be determined? Injury. 2000;31:421-4.

16. Mitani S, Shimizu M, Abo M, Hagino H, Kurozawa Y. Risk factors for second hip fractures among elderly patients. $J$ Orthop Sci. 2010;15:192-7. 